\title{
ON ALMOST NEARLY CONTINUOUS FUNCTIONS WITH REFERENCE TO MULTIFUNCTIONS
}

\author{
ANDRZEJ RYCHLEWICZ
}

\begin{abstract}
We deal with a new kind of continuity of multifunctions, namely almost nearly quasi-continuous multifunctions have been introduced. Several properties of almost nearly quasi-continuous and almost upper (lower) nearly quasicontinuous multifunctions have been obtained.
\end{abstract}

\section{Introduction}

Many mathematicians have been investigating a lot of forms of continuity. In 2004, the notion of almost nearly continuity of multifunctions was introduced by Ekici and it was shown (see EK]) that this notion is closely related to nearly compact spaces, especially to N-closed sets. The class of nearly compact spaces was introduced by M. K. Singal and Asha Matur in 1969 (see [SM]) while N-closed sets were defined by D. C a rn a ha $\mathrm{n}$ in [CA]. One kind of a "weak" continuity is a quasi-continuity. It seems to be natural to consider almost nearly quasi-continuous multifunctions. So, the purpose of the present paper is to introduce and study this kind of continuity.

\section{Preliminaries}

From now on, $\operatorname{cl}(A)$ and int $(A)$ will represent the closure and interior of a subset $A$ of a topological space, respectively.

A subset of a topological space is said to be regularly open (closed) if it is the interior of some closed set (the closure of some open set) or, equivalently, if it is the interior of its own closure (the closure of its own interior) (see, for instance, [SM]). A set $A$ in a topological space will be called semi-open (semi-closed) if $A \subset \operatorname{cl}(\operatorname{int}(A))(A \subset \operatorname{int}(\operatorname{cl}(A)))$.

2000 Mathematics Subject Classification: 54A10, 54C08, 54C50, 54C60.

Keywords: nearly quasi-continuous multifunction, nearly compact space, N-closed set, multifunction, Baire's category, almost coN-closed topology. 


\section{ANDRZEJ RYCHLEWICZ}

It is known that the union of arbitrary class of semi-open sets is semi-open. The notion of semi-open sets and their properties were introduced and studied by $\mathrm{N}$. L e vin e $[\mathrm{LE}]$.

A topological space is called nearly compact if for every open cover there is a finite subclass, such that the interiors of the closures of its members cover the space or, equivalently, if every cover of the space by regularly open sets has a finite subcover (see $[\mathrm{SM}]$ ). A subset $A$ of a topological space is called N-closed if for any cover $\mathcal{C}$ of $A$ by open sets there exists a finite subclass $\tilde{\mathcal{C}}$ such that the interiors of the closures of the set from $\tilde{\mathcal{C}}$ covers $A$ or, equivalently, if for any cover of $A$ by regularly open sets, there exists a finite subcover (see [CA]).

Next, $(Y, \tau)$ will denote a topological space. We will often use the following fact.

LemMA 1. Let $G$ be an open subset of a topological space $Y$ such that $Y \backslash G$ is an $N$-closed set. Then $\operatorname{int}(\mathrm{cl}(G))$ is a regularly open set having an $N$-closed complement.

Proof. It is clear that the set $\operatorname{int}(\operatorname{cl}(G))$ is regularly open. Let us denote $A=Y \backslash \operatorname{int}(\operatorname{cl}(G))$. Of course, $A \subset Y \backslash G$. Let $\left\{G_{t}\right\}_{t \in T}$ be an open cover of the set $A$. Then $\left\{G_{t}\right\}_{t \in T} \cup(Y \backslash A)$ is an open cover of the set $Y \backslash G$. Then there exist indexes $t_{1}, \ldots, t_{k}$ such that

$$
\bigcup_{i=1}^{k} \operatorname{int}\left(\operatorname{cl}\left(G_{t_{i}}\right)\right) \cup \operatorname{int}(\operatorname{cl}(Y \backslash A)) \supset Y \backslash G \text {. }
$$

Since

$$
\operatorname{int}(\operatorname{cl}(Y \backslash A))=\operatorname{int}(\operatorname{cl}(\operatorname{int}(\operatorname{cl}(G))))=\operatorname{int}(\operatorname{cl}(G))
$$

then

$$
A \cap \operatorname{int}(\operatorname{cl}(Y \backslash A))=\emptyset
$$

It was shown that

$$
\bigcup_{i=1}^{k} \operatorname{int}\left(\operatorname{cl}\left(G_{t_{i}}\right)\right) \supset A .
$$

The proof of the N-closedness of the set $A$ is finished.

Let $\tau^{R O}$ be a collection of subsets of $Y$ defined as follows.

$$
\tau^{R O}=\{U \in \tau: U \text { is regularly open set and } Y \backslash U \text { is N-closed }\} .
$$

The collection $\tau^{R O}$ forms a base of some topology $\tau^{N}$ in $Y$ called almost coN-closed topology of the topology $\tau$ (see [KR]). The topology $\tau_{s}$, a base of which is equal to the family of all regularly open sets in the topology $\tau$, is called semi-regularization of the topology $\tau$ (see $[\mathrm{PT}]$ ). 


\section{ON ALMOST NEARLY CONTINUOUS FUNCTIONS}

From now on, we will assume that $X$ is a topological space and $F: X \rightarrow Y$ is a multifunction, i.e., $F(x)$ is a nonempty subset of $Y$ for each $x$ from $X$.

$F$ is said to be almost nearly continuous at a point $x \in X$ if for any open subsets $V_{1}$ and $V_{2}$ of $Y$ having $N$-closed complement such that

$$
x \in F^{+}\left(V_{1}\right) \cap F^{-}\left(V_{2}\right)
$$

there exists an open neighborhood $U$ of $x$ such that

$$
U \subset F^{+}\left(\operatorname{int}\left(\operatorname{cl}\left(V_{1}\right)\right)\right) \cap F^{-}\left(\operatorname{int}\left(\operatorname{cl}\left(V_{2}\right)\right)\right) .
$$

If $F$ is almost nearly continuous at any $x \in X$, it is called to be almost nearly continuous.

Let us recall the notions of semi-continuous (lower and upper), continuous, quasi-continuous (lower and upper) and quasi-continuous multifunctions.

$F$ is said to be upper (lower) semi-continuous at a point $x \in X$ if for any open subset $V$ of $Y$ such that $x \in F^{+}(V)\left(x \in F^{-}(V)\right)$ there exists an open neighborhood $U$ of $x$ such that $U \subset F^{+}(V)\left(U \subset F^{-}(V)\right)$.

$F$ is semi-continuous at a point $x \in X$ if for any open subsets $V_{1}$ and $V_{2}$ of $Y$ such that $x \in F^{+}\left(V_{1}\right) \cap F^{-}\left(V_{2}\right)$ there exists an open neighborhood set $U$ of $x$ such that

$$
U \subset F^{+}\left(V_{1}\right) \cap F^{-}\left(V_{2}\right) .
$$

$F$ is called upper (lower) quasi-continuous at a point $x \in X$ (see [NE]) if for any open subset $V$ of $Y$ such that $x \in F^{+}(V)\left(x \in F^{-}(V)\right)$ and for any open neighborhood $U$ of $x$ there exists a nonempty open set $W \subset U$ such that

$$
W \subset F^{+}(V) \quad\left(W \subset F^{-}(V)\right) .
$$

We say that $F$ is quasi-continuous at a point $x \in X$ (see [NE]) if for any open subsets $V_{1}$ and $V_{2}$ of $Y$ such that $x \in F^{+}\left(V_{1}\right) \cap F^{-}\left(V_{2}\right)$ and for any open neighborhood $U$ of $x$ there exists a nonempty open set $W \subset U$ such that $U \subset F^{+}\left(V_{1}\right) \cap F^{-}\left(V_{2}\right)$.

A multifunction $F$ is said to be semi-continuous (lower and upper), continuous, quasi-continuous (lower and upper) and quasi-continuous multifunctions if $F$ has this property at any point of $X$.

A multifunction $F: X \rightarrow Y$ is said to be upper (lower) almost nearly continuous at a point $x \in X$ if for any open subset $V$ of $Y$ having $N$-closed complement such that $x \in F^{+}(V)\left(x \in F^{-}(V)\right)$ there exists an open neighborhood $U$ of $x$ such that

$$
U \subset F^{+}(\operatorname{int}(\operatorname{cl}(V))), \quad U \subset F^{-}(\operatorname{int}(\operatorname{cl}(V))) .
$$

We say that a multifunction $F$ is upper (lower) almost nearly continuous if it is upper (lower) almost nearly continuous at any point $x \in X$ (see [EK]).

Now, we are ready to introduce the notions of an almost nearly quasi-continuous and upper (lower) almost nearly quasi-continuous multifunction. 


\section{ANDRZEJ RYCHLEWICZ}

A multifunction $F$ is called upper (lower) almost nearly quasi-continuous at a point $x \in X$ if for any open subset $V$ of $Y$ having $N$-closed complement such that $x \in F^{+}(V)\left(x \in F^{-}(V)\right)$ and for any open set $U \ni x$ there exists a nonempty open set $W \subset U$ such that

$$
W \subset F^{+}(\operatorname{int}(\operatorname{cl}(V))), \quad W \subset F^{-}(\operatorname{int}(\operatorname{cl}(V))) .
$$

We call a multifunction $F$ almost nearly quasi-continuous at a point $x \in X$ if for any open subsets $V_{1}$ and $V_{2}$ of $Y$ having $N$-closed complement such that $x \in F^{+}\left(V_{1}\right) \cap F^{-}\left(V_{2}\right)$ and for any open set $U \ni x$ there exists a nonempty open set $W \subset U$ such that

$$
W \subset F^{+}(\operatorname{int}(\operatorname{cl}(V))) \cap F^{-}(\operatorname{int}(\operatorname{cl}(V))) .
$$

A multifunction $F$ is said to be an almost nearly quasi-continuous (upper almost nearly quasi-continuous, lower almost nearly quasi-continuous) multifunction if $F$ has this property at any point of $X$.

By $D_{n}^{u}(F), D_{n}^{l}(F)$, and $D_{n}(F)$ we will denote the set of all points at which the multifunction $F$ is not upper, not lower and not almost nearly continuous, respectively.

By $Q C_{n}(F), Q C_{n}^{u}(F), Q C_{n}^{l}(F), Q C(F), Q C^{u}(F)$ and $Q C^{l}(F)$, respectively, we will denote the set of all points at which a multifunction $F$ is almost nearly quasi-continuous, upper almost nearly quasi-continuous, lower almost nearly quasi-continuous, quasi-continuous, upper quasi-continuous, lower quasi-continuous, respectively.

\section{Basic properties}

In this section, a lot of equivalence conditions to the ones introduced in the definitions of an almost nearly quasi-continuous and upper (lower) almost nearly quasi-continuous multifunction will be given and proved.

TheOREM 1. Let $F: X \rightarrow Y$ be a multifunction. The following statements are equivalent.

(a) F is upper almost nearly quasi-continuous.

(b) For any $x \in X$ and for any regularly open set $G \subset Y$ having $N$-closed complement such that $F(x) \subset G$ and for any open set $U \ni x$, there exists a nonempty open set $W \subset U$ such that $F(z) \subset G$ for any $z \in W$.

(c) For any $x \in X$, any closed $N$-closed set $K \subset Y$ such that $x \in F^{+}(Y \backslash K)$ and for any closed set $H$ such that $x \in X \backslash H$, there exists a closed set $M \supset H, M \neq X$ such that $F^{-}(\operatorname{cl}(\operatorname{int}(K))) \subset M$. 


\section{ON ALMOST NEARLY CONTINUOUS FUNCTIONS}

(d) For any $x \in X$ and for any open set $G \subset Y$ having $N$-closed complement such that $F(x) \subset G$, there exists a semi-open set $A \ni x$ such that

$$
A \subset F^{+}(\operatorname{int}(\operatorname{cl}(G))) .
$$

(e) $F^{+}(G)$ is semi-open set for any regularly open set $G \subset Y$ having $N$-closed complement.

(f) $F^{-}(K)$ is semi-closed set for any regularly closed $N$-closed set $K \subset Y$.

P r o of. $(a) \Rightarrow(b)$. Let $x \in X$ and let $G$ be a regularly open subset of $Y$ having N-closed complement such that $F(x) \subset G$ and let $U \ni x$ be an open subset of $X$. Under the assumptions ( $F$ is upper almost nearly continuous), there exists an open nonempty set $W \subset U$ such that $W \subset F^{+}(\operatorname{int}(\operatorname{cl}(G))) . G$ is regularly open, then $G=\operatorname{int}(\operatorname{cl}(G))$ and, consequently, $W \subset F^{+}(G)$.

$(b) \Rightarrow(a)$. Let now $x \in X$ and let $G$ be an open set having N-closed complement such that $F(x) \subset G$ and let $U \ni x$ be an open subset of $X$. By Lemma 1, we know that the set int $(\operatorname{cl}(G))$ is regularly open and $Y \backslash \operatorname{int}(\operatorname{cl}(G))$ is N-closed. Since $F(x) \subset \operatorname{int}(\operatorname{cl}(G))$ then there exists an open nonempty set $W \subset U$ such that $W \subset F^{+}(\operatorname{int}(\operatorname{cl}(G)))$.

$(a) \Rightarrow(c)$. Let $x \in X$ and let $K$ be a closed N-closed subset of $Y$ such that $x \in F^{+}(Y \backslash K)$. It is clear that $Y \backslash K$ is an open subset of $Y$ having N-closed complement. Let $H$ be a closed subset of $Y$ such that $x \in X \backslash H$. Then $X \backslash H$ is an open set. According to the definition of upper almost nearly continuity, there exists an open nonempty set $W \subset X \backslash H$ such that

Let us observe that

$$
W \subset F^{+}(\operatorname{int}(\operatorname{cl}(Y \backslash K))) \text {. }
$$

$$
\operatorname{int}(\operatorname{cl}(Y \backslash K))=\operatorname{int}(Y \backslash \operatorname{int}(K))=Y \backslash \operatorname{cl}(\operatorname{int}(K)) .
$$

It follows that

$$
W \subset F^{+}\left(Y \backslash(\operatorname{cl}(\operatorname{int}(K)))=X \backslash F^{-}(\operatorname{cl}(\operatorname{int}(K))) .\right.
$$

Let $M=X \backslash W$, then

$$
X \backslash M \subset X \backslash F^{-}(\operatorname{cl}(\operatorname{int}(K)))
$$

since $M \supset F^{-}(\operatorname{cl}(\operatorname{int}(K)))$. It is evident that $M$ is a closed set and $M \neq X$.

$(c) \Rightarrow(a)$. Let $x \in X$ and let $G$ be an open subset of $Y$ having N-closed complement and let $F(x) \subset G$. Therefore, $K=Y \backslash G$ is a closed N-closed subset of $Y$ such that $x \in F^{+}(Y \backslash K)$. Let $U \ni x$ be an open subset of $X$. Then $H=X \backslash U$ is a closed set such that $x \in X \backslash H$. Under the assumptions there exists a closed set $M \supset H, M \neq X$ such that $F^{-}(\operatorname{cl}(\operatorname{int}(K))) \subset M$. The last inclusion implies that $X \backslash F^{+}(\operatorname{int}(\operatorname{cl}(G))) \subset M=X \backslash W$, where $W=X \backslash M$ 


\section{ANDRZEJ RYCHLEWICZ}

is an open nonempty set. It was shown that $W \subset F^{+}(\operatorname{int}(\operatorname{cl}(G)))$. It is easy to see that $W \subset U$.

$(a) \Rightarrow(d)$. Let $x \in X$ and let $G$ be an open subset of $Y$ having N-closed complement and let $F(x) \subset G$. We know that for any open neighborhood $U$ of the point $x$ there exists an open nonempty set $W_{U} \subset U$ such that

$$
W_{U} \subset F^{+}(\operatorname{int}(\operatorname{cl}(G))) \text {. }
$$

Let $A=\{x\} \cup \bigcup\left\{W_{U}: U\right.$ is an open neighbourhood of $\left.x\right\}$. Hence

$$
A \subset \operatorname{cl}(\operatorname{int}(A))
$$

and, consequently, $A \ni x$ is a semi-open set.

Additionally, $A \subset F^{+}(\operatorname{int}(\operatorname{cl}(G)))$.

$(d) \Rightarrow(a)$. Let $x \in X$ and let $G$ be an open subset of $Y$ having N-closed complement and let $F(x) \subset G$. Let $U \ni x$ be an open subset of $X$. Under assumptions, there exists a semi-open set $A \ni x$ such that $A \subset F^{+}(\operatorname{int}(\operatorname{cl}(G)))$. Let $W=U \cap \operatorname{int}(A)$.

Because of $U \cap A \neq \emptyset, W \neq \emptyset$. It is easy to check that $W \subset U$ and $W \subset A$. Therefore $W \subset F^{+}(\operatorname{int}(\operatorname{cl}(G)))$.

$(d) \Rightarrow(e)$. Let $G$ be a regularly open subset of $Y$ having N-closed complement and let $x \in F^{+}(G)$. Then $F(x) \subset G$. Under assumptions, there exists a semiopen set $A_{x} \ni x$ such that

$$
A_{x} \subset F^{+}(G)=F^{+}(\operatorname{int}(\operatorname{cl}(G))) .
$$

It can be easily seen that the set $A=\bigcup\left\{A_{x}: x \in F^{+}(G)\right\}$ is semi-open and equal to the set $F^{+}(G)$.

$(e) \Rightarrow(d)$. Let $x \in X$ and $G$ be an open subset of $Y$ having N-closed complement such that $F(x) \subset G$. Then, int $(\operatorname{cl}(G))$ is a regularly open set having N-closed complement. Therefore, $F^{+}(\operatorname{int}(\mathrm{cl}(G)))$ is semi-open. Of course, $x \in F^{+}(\operatorname{int}(\operatorname{cl}(G)))$.

$(e) \Rightarrow(f)$. Let $K$ be a regularly closed N-closed subset of $Y$. Then $Y \backslash K$ is a regularly open having $\mathrm{N}$-closed complement subset of $Y$. Under assumptions, $F^{+}(Y \backslash K)$ is a semi-open set. From this, we see that the set $X \backslash F^{+}(Y \backslash K)=$ $F^{-}(K)$ is semi-closed.

$(f) \Rightarrow(e)$. The proof is similar to the previous case.

Theorem 2. Let $F: X \rightarrow Y$ be a multifunction. The following statements are equivalent.

(a) F is lower almost nearly quasi-continuous.

(b) For any $x \in X$ and for any regularly open set $G \subset Y$ having $N$-closed complement such that $F(x) \cap G \neq \emptyset$ and for any open set $U \ni x$, there exists a nonempty open set $W \subset U$ such that $F(z) \cap G \neq \emptyset$ for any $z \in W$. 
(c) For any $x \in X$ and for any closed $N$-closed set $K \subset Y$ such that $x \in$ $F^{-}(Y \backslash K)$ and for any closed set $H$ such that $x \in X \backslash H$, there exists a closed set $M \supset H, M \neq X$ such that $F^{+}(\operatorname{cl}(\operatorname{int}(K))) \subset M$.

(d) For any $x \in X$ and for any open set $G \subset Y$ having $N$-closed complement such that $F(x) \cap G \neq \emptyset$, there exists a semi-open set $A \ni x$ such that $A \subset F^{-}(\operatorname{int}(\operatorname{cl}(G)))$.

(e) The set $F^{-}(G)$ is semi-open for any regularly open set $G \subset Y$ having $N$-closed complement.

( $f$ ) The set $F^{+}(K)$ is semi-closed for any regularly closed $N$-closed set $K \subset Y$.

Theorem 3. Let $F: X \rightarrow Y$ be a multifunction. The following statements ere equivalent.

(a) $F$ is almost nearly quasi-continuous.

(b) For any $x \in X$ and for any regularly open subsets $G_{1}, G_{2}$ of $Y$ having $N$-closed complement such that $F(x) \subset G_{1}$ and $F(x) \cap G_{2} \neq \emptyset$ and for any open set $U \ni x$, there exists a nonempty open set $W \subset U$ such that $F(z) \subset G_{1}$ and $F(z) \cap G_{2} \neq \emptyset$ for any $z \in W$.

(c) For any $x \in X$ and for any closed $N$-closed sets $K_{1}, K_{2} \subset Y$ such that $x \in F^{+}\left(Y \backslash K_{1}\right) \cap F^{-}\left(Y \backslash K_{2}\right)$ and for any closed set $H$ such that $x \in X \backslash H$, there exists a closed set $M \supset H, M \neq X$ such that $F^{-}\left(\operatorname{cl}\left(\operatorname{int}\left(K_{1}\right)\right)\right) \cup$ $F^{+}\left(\operatorname{cl}\left(\operatorname{int}\left(K_{2}\right)\right)\right) \subset M$.

(d) For any $x \in X$ and for any open sets $G_{1}, G_{2} \subset Y$ having $N$-closed complement such that $F(x) \subset G_{1}$ and $F(x) \cap G_{2} \neq \emptyset$, there exists a semi-open set $A \ni x$ such that $A \subset F^{+}\left(\operatorname{int}\left(\operatorname{cl}\left(G_{1}\right)\right)\right) \cap F^{-}\left(\operatorname{int}\left(\operatorname{cl}\left(G_{2}\right)\right)\right)$.

(e) The set $F^{+}\left(G_{1}\right) \cap F^{-}\left(G_{2}\right)$ is semi-open for any regularly open sets $G_{1}, G_{2}$ of $Y$ having $N$-closed complement.

( $f$ ) The set $F^{-}\left(K_{1}\right) \cup F^{+}\left(K_{2}\right)$ is semi-closed for any regularly closed $N$-closed set $K_{1}, K_{2} \subset Y$.

Pr o of. $(a) \Rightarrow(b)$. Let $x \in X$ and $G_{1}, G_{2}$ be regularly open subsets of $Y$ having N-closed complement such that $x \in F^{+}\left(G_{1}\right) \cap F^{-}\left(G_{2}\right)$. Let $U$ be an open subset of $X$ containing $x$. Under assumption, there exists a nonempty open set $W \subset U$ such that $W \subset F^{+}\left(\operatorname{int}\left(\operatorname{cl}\left(G_{1}\right)\right)\right) \cap F^{-}\left(\operatorname{int}\left(\operatorname{cl}\left(G_{2}\right)\right)\right)$. Hence,

$$
W \subset F^{+}\left(G_{1}\right) \cap F^{-}\left(G_{2}\right)
$$

because $G_{1}, G_{2}$ are regularly open sets.

$(b) \Rightarrow(a)$. Let $x \in X$ and $G_{1}, G_{2}$ be open sets having N-closed complement such that $x \in F^{+}\left(G_{1}\right) \cap F^{-}\left(G_{2}\right)$. Then, by Lemma 1, int $\left(\operatorname{cl}\left(G_{1}\right)\right)$, int $\left(\operatorname{cl}\left(G_{2}\right)\right)$ are regularly open sets and it is clear that

$$
x \in F^{+}\left(\operatorname{int}\left(\operatorname{cl}\left(G_{1}\right)\right)\right) \cap F^{-}\left(\operatorname{int}\left(\operatorname{cl}\left(G_{2}\right)\right)\right) .
$$


Then, for any open set $U \ni x$, there exists an open nonempty set $W \subset U$ such that $W \subset F^{+}\left(\operatorname{int}\left(\operatorname{cl}\left(G_{1}\right)\right)\right) \cap F^{-}\left(\operatorname{int}\left(\operatorname{cl}\left(G_{2}\right)\right)\right)$.

$(a) \Rightarrow(c)$. Let $x \in X$ and $K_{1}, K_{2} \subset Y$ be closed N-closed sets such that

$$
x \in F^{+}\left(Y \backslash K_{1}\right) \cap F^{-}\left(Y \backslash K_{2}\right) .
$$

Let $H$ be a closed subset of $X$ such that $x \in X \backslash H$. Then, there exists an open nonempty set $W \subset U=X \backslash H$ such that

$$
W \subset F^{+}\left(\operatorname{int}\left(\operatorname{cl}\left(Y \backslash K_{1}\right)\right)\right) \cap F^{-}\left(\operatorname{int}\left(\operatorname{cl}\left(Y \backslash K_{2}\right)\right)\right) .
$$

Let us denote $M=X \backslash W$. Then, $M$ is a closed set different from $X$ and

$$
\begin{aligned}
M & \supset X \backslash\left[F^{+}\left(\operatorname{int}\left(\operatorname{cl}\left(Y \backslash K_{1}\right)\right)\right) \cap F^{-}\left(\operatorname{int}\left(\operatorname{cl}\left(Y \backslash K_{2}\right)\right)\right)\right] \\
& =\left[X \backslash F^{+}\left(\operatorname{int}\left(\operatorname{cl}\left(Y \backslash K_{1}\right)\right)\right)\right] \cup\left[X \backslash F^{-}\left(\operatorname{int}\left(\operatorname{cl}\left(Y \backslash K_{2}\right)\right)\right)\right] \\
& =F^{-}\left(\operatorname{cl}\left(\operatorname{int}\left(K_{1}\right)\right)\right) \cup F^{+}\left(\operatorname{cl}\left(\operatorname{int}\left(K_{2}\right)\right)\right) .
\end{aligned}
$$

$(c) \Rightarrow(a)$. The proof is similar to the previous one.

$(a) \Rightarrow(d)$. Let $x \in X$ and $G_{1}, G_{2}$ be open subsets of $Y$ having N-closed complement such that $F(x) \subset G_{1}$ and $F(x) \cap G_{2} \neq \emptyset$. We know that for any open subset $U \ni x$ of $Y$ there exists a nonempty open set $W_{U} \subset U$ such that $W_{U} \subset F^{+}\left(\operatorname{int}\left(\operatorname{cl}\left(G_{1}\right)\right)\right) \cap F^{-}\left(\operatorname{int}\left(\operatorname{cl}\left(G_{2}\right)\right)\right)$. Let

$$
A=\{x\} \cup \bigcup\left\{W_{U}: U \text { is an open neighbourhood of } x\right\} .
$$

It is clear that $A \subset \operatorname{cl}(\operatorname{int}(A))$ and hence semi-open. Of course, $x \in A$ and $A \subset F^{+}\left(\operatorname{int}\left(\operatorname{cl}\left(G_{1}\right)\right)\right) \cap F^{-}\left(\operatorname{int}\left(\operatorname{cl}\left(G_{2}\right)\right)\right)$.

$(d) \Rightarrow(a)$. Let $x \in X$ and $G_{1}, G_{2}$ be open subsets of $Y$ having N-closed complement such that $x \in F^{+}\left(G_{1}\right) \cap F^{-}\left(G_{2}\right)$. Let $U$ be an open neighborhood of $x$. We know that there exists a semi open set

$$
A \subset F^{+}\left(\operatorname{int}\left(\operatorname{cl}\left(G_{1}\right)\right)\right) \cap F^{-}\left(\operatorname{int}\left(\operatorname{cl}\left(G_{2}\right)\right)\right)
$$

such that $x \in A$. Let $W$ denote the set $U \cap \operatorname{int}(A)$. Since $U \cap A \neq \emptyset$, then $W \neq \emptyset$. Of course, $W \subset U$ and $W \subset A$. Therefore,

$$
W \subset F^{+}\left(\operatorname{int}\left(\operatorname{cl}\left(G_{1}\right)\right)\right) \cap F^{-}\left(\operatorname{int}\left(\operatorname{cl}\left(G_{2}\right)\right)\right) .
$$

$(d) \Rightarrow(e)$. Let $G_{1}, G_{2}$ be regularly open subsets of $Y$ having N-closed complement such that $x \in F^{+}\left(G_{1}\right) \cap F^{-}\left(G_{2}\right)$. Let us denote by $A_{x}$ a semi-open set such that

$$
x \in A_{x} \subset F^{+}\left(\operatorname{int}\left(\operatorname{cl}\left(G_{1}\right)\right)\right) \cap F^{-}\left(\operatorname{int}\left(\operatorname{cl}\left(G_{2}\right)\right)\right)=F^{+}\left(G_{1}\right) \cap F^{-}\left(G_{2}\right) .
$$




\section{ON ALMOST NEARLY CONTINUOUS FUNCTIONS}

Then, the set $A=\bigcup_{x \in F^{+}\left(G_{1}\right) \cap F^{-}\left(G_{2}\right)} A_{x}$ is semi-open and equal to the set

$$
F^{+}\left(G_{1}\right) \cap F^{-}\left(G_{2}\right) \text {. }
$$

$(e) \Rightarrow(d)$. Let $x \in X$ and $G_{1}, G_{2}$ be open subsets of $Y$ having N-closed complement such that $F(x) \in G_{1}$ and $F(x) \cap G_{2} \neq \emptyset$. Then by Lemma 1, int $\left(\mathrm{cl}\left(G_{1}\right)\right)$ and int $\left(\mathrm{cl}\left(G_{2}\right)\right)$ are regularly open sets having N-closed complement and $F(x) \subset \operatorname{int}\left(\operatorname{cl}\left(G_{1}\right)\right)$ and $F(x) \cap \operatorname{int}\left(\operatorname{cl}\left(G_{2}\right)\right) \neq \emptyset$. Under assumption, $F^{+}\left(\operatorname{int}\left(\operatorname{cl}\left(G_{1}\right)\right)\right) \cap F^{-}\left(\operatorname{int}\left(\operatorname{cl}\left(G_{2}\right)\right)\right)$ is a semi-open set. According to the above remark, the proof of this part is completed.

$(e) \Rightarrow(f)$. Let $K_{1}, K_{2}$ be regularly closed N-closed subsets of $Y$. Then the set

$$
F^{+}\left(Y \backslash K_{1}\right) \cap F^{-}\left(Y \backslash K_{2}\right)
$$

is semi-open. The complement of this set is semi-closed and is equal to the set

$$
X \backslash\left[F^{+}\left(Y \backslash K_{1}\right) \cap F^{-}\left(Y \backslash K_{2}\right)\right]=F^{-}\left(K_{1}\right) \cup F^{+}\left(K_{2}\right) .
$$

$(f) \Rightarrow(e)$. The proof is similar to the previous one.

\section{Main results}

In the papers [EL] and [EN] the following theorems were proved.

Theorem 4. Let $(Y, \tau)$ be a second countable topological space.

(a) If $F: X \rightarrow Y$ is upper quasi-continuous multifunction such that $F(x)$ is a compact subset of the space $Y$ for any $x \in X$, then the set of all points at which $F$ is not upper semicontinuous is of the first Baire's category see [EL, Th. 15].

(b) If $F: X \rightarrow Y$ is lower quasi-continuous multifunction then the set of all points at which $F$ is not lower semicontinuous is of the first Baire's category see [EL, Th. 16].

Theorem 5. Let $(Y, \tau)$ be a second countable topological space. If $F: X \rightarrow Y$ is a multifunction such that $F(x)$ is a compact subset of the space $Y$ for any $x \in X$, then the set $Q C^{u}(F) \cap Q C^{l}(F) \backslash Q C(F)$ is of the first Baire's category see [EN, Th. 1].

The question: Are the analogous theorems for almost near continuity case true? The answer is positive. As it turns out, it is very useful to transfer our investigations to the multifunctions $F: X \rightarrow\left(Y, \tau^{N}\right)$ and to consider an almost coN-closed topology. First, we need two lemmas. 


\section{ANDRZEJ RYCHLEWICZ}

Lemma 2. A multifunction $F: X \rightarrow(Y, \tau)$ is almost nearly continuous (upper almost nearly continuous, lower almost nearly continuous) at a point $x \in X$ if and only if the multifunction $F: X \rightarrow\left(Y, \tau^{N}\right)$ is continuous (upper semicontinuous, lower semi-continuous) at a point $x$.

Pr o of. We know that $F$ is upper (lower) almost nearly continuous at a point $x \in X$ if and only if for any regularly open set $V$ having N-closed complement such that $x \in F^{+}(V)\left(x \in F^{-}(V)\right)$ there exists an open set $U$ containing $x$ such that

$$
U \subset F^{+}(V) \quad\left(U \subset F^{+}(V)\right)
$$

see [EK, Th. 3.(3) and Th. 6.(3)]. Due to these facts the proof of upper (lower) case is evident.

In order to prove almost nearly continuous case, let us assume that $F: X \rightarrow$ $(Y, \tau)$ is almost nearly continuous at some point $x$. Let $V_{1}, V_{2}$ be regularly open sets having $\mathrm{N}$-closed complement such that $F(x) \subset V_{1}$ and $F(x) \cap V_{2} \neq \emptyset$. Under the assumptions, there exists an open set $U \ni x$ such that

$$
U \subset F^{+}\left(\operatorname{int}\left(\operatorname{cl}\left(V_{1}\right)\right)\right) \cap F^{-}\left(\operatorname{int}\left(\operatorname{cl}\left(V_{2}\right)\right)\right) .
$$

Since $V_{1}, V_{2}$ are regularly open sets, the last condition can be written as $U \subset$ $F^{+}\left(V_{1}\right) \cap F^{-}\left(V_{2}\right)$.

Now, let us assume that $F: X \rightarrow\left(Y, \tau^{N}\right)$ is a continuous multifunction and let $V_{1}, V_{2}$ be open sets having $\mathrm{N}$-closed complement such that $x \in F^{+}\left(V_{1}\right)$ and $x \in$ $F^{-}\left(V_{2}\right)$. By Lemma 1, the sets int $\left(\mathrm{cl}\left(V_{1}\right)\right)$ and int $\left(\operatorname{cl}\left(V_{2}\right)\right)$ are regularly open sets having N-closed complement and $x \in F^{+}\left(\operatorname{int}\left(\operatorname{cl}\left(V_{1}\right)\right)\right) \cap F^{-}\left(\operatorname{int}\left(\operatorname{cl}\left(V_{2}\right)\right)\right)$. Thus

$$
U \subset F^{+}\left(\operatorname{int}\left(\operatorname{cl}\left(V_{1}\right)\right)\right) \cap F^{-}\left(\operatorname{int}\left(\operatorname{cl}\left(V_{2}\right)\right)\right)
$$

for some open set $U \ni x$.

The following lemma can be proved in a similar way.

Lemma 3. A multifunction $F: X \rightarrow(Y, \tau)$ is almost nearly quasi-continuous (upper almost nearly quasi-continuous, lower almost nearly quasi-continuous) at a point $x \in X$ if and only if the multifunction $F: X \rightarrow\left(Y, \tau^{N}\right)$ is quasicontinuous (upper quasi-continuous, lower quasi-continuous) at a point $x$.

Now, we can start to formulate and prove the main theorems.

THEOREM 6. Let us assume that there exists a countable base of the almost coN-closed topology $\tau^{N}$ of a topology $\tau$.

(a) If $F: X \rightarrow(Y, \tau)$ is upper almost nearly quasi-continuous multifunction such that $F(x)$ is $N$-closed subset of the space $Y$ for any $x \in X$, then the set $D_{n}^{u}(F)$ is of the first Baire's category. 
(b) If $F: X \rightarrow(Y, \tau)$ is lower almost nearly quasi-continuous multifunction then the set $D_{n}^{l}(F)$ is of the first Baire's category.

Proof.

(a) Under the assumption, $\left(Y, \tau^{N}\right)$ is a second countable space. By Lemma 3 $F: X \rightarrow\left(Y, \tau^{N}\right)$ is upper quasi-continuous multifunction. Let $x$ be an arbitrary point of $X$. Because the set $F(x)$ is $\mathrm{N}$-closed with respect to topology $\tau$, then $F(x)$ is a compact subset of the topological space $\left(Y, \tau_{s}\right)$ see [NO, Th. 3.1.]. Since the topology $\tau^{N}$ is weaker than the topology $\tau_{s}$, the last condition means that $F(x)$ is a compact subset of the topological space $\left(Y, \tau^{N}\right)$ for any $x \in X$. By Lemma 2, the set $D_{n}^{u}(F)$ is equal to the set of all points at which a multifunction $F: X \rightarrow\left(Y, \tau^{N}\right)$ is not upper semicontinuous. It follows that the set $D_{n}^{u}(F)$ is of the first Baire's category (see Th. 4(a)).

(b) The proof is similar to the previous case.

TheOREM 7. Let us assume that there exists a countable base of the almost coN-closed topology $\tau^{N}$ of a topology $\tau$. If $F: X \rightarrow(Y, \tau)$ is a multifunction such that $F(x)$ is $N$-closed subset of the space $Y$ for any $x \in X$, then the set $Q C_{n}^{u}(F) \cap Q C_{n}^{l}(F) \backslash Q C_{n}(F)$ is of the first Baire's category.

Proof. Similarly as in the proof of Theorem 6. N-closedness of the set $F(x)$ with respect to the topology $\tau$, implies a compactness of $F(x)$ in the topological space $\left(Y, \tau^{N}\right)$, for any $x \in X$. By Lemma 3, we have the following equalities

$$
Q C_{n}(F)=Q C(F), \quad Q C_{n}^{u}(F)=Q C^{u}(F) \quad \text { and } \quad Q C_{n}^{l}(F)=Q C^{l}(F) .
$$

Since $Q C^{u}(F) \cap Q C^{l}(F) \backslash \cap Q C(F)$ is of the first Baire's category (see Th. 51), then the set $Q C_{n}^{u}(F) \cap Q C_{n}^{l}(F) \backslash Q C_{n}(F)$ is of the first Baire's category.

\section{REFERENCES}

[CA] CARNAHAN, D.: Locally nearly-compact spaces, Boll. Unione Mat. Ital., IV. Ser. 6 (1972), 146-153.

[EK] EKICI, E.: Almost nearly continuous multifunctions, Acta Math. Univ. Comenianae, LXXIII (2004), 175-186.

[EL] EWERT, J.-LIPSKI, T.: Quasi-continuous multivalued mappings, Math. Slovaca 33 (1983), 69-74.

[EN] EWERT, J.-NEUBRUNN, T.: On quasi-continuous multivalued maps, Demonstratio Math. XXI (1988), 697-711.

[KR] KONSTADILAKI-SAVVOPOULOU, CH.-REILLY, I. L.: On almost-N-continuous functions, J. Austral. Math. Soc., Ser. A 59 (1995), 118-130.

[LE] LEVINE, N.: Semi-open sets and semi-continuity in topological spaces, Amer. Math. Monthly $\mathbf{7 0}$ (1963), 36-43. 


\section{ANDRZEJ RYCHLEWICZ}

[NE] NEUBRUNN, T.: Quasi-continuity, Real Anal. Exchange 14 (1989), 259-306.

[NO] NOIRI, T.: Remarks on locally nearly-compact spaces, Boll. Unione Mat. Ital., IV. Ser. 10 (1974), 36-43.

[PT] PORTER, J. R.-THOMAS, J.: On H-closed and minimal Hausdorff spaces, Trans. Amer. Math. Soc. 138 (1969), 159-170.

[SM] SINGAL, M. K.-MATHUR, A.: On nearly compact spaces, Boll. Unione Mat. Ital., IV. Ser. 2 (1969), 702-710.

Received September 24, 2007

Faculty of Mathematics and Informatics Eódź University

ul. Stefana Banacha 22

PL-90-238 Eódź

POLAND

E-mail: anrychle@math.uni.lodz.pl 